\title{
Der verdrängte Keynes
}

\author{
In diesen Tagen jährt sich der Tod von John Maynard Keynes zum 75. Mal. Er war einer der \\ bedeutendsten Ökonomen des 20. Jahrhunderts, wenn nicht gar der bedeutendste überhaupt. \\ Dabei waren seine Lehren und seine Ratschläge schon zu seinen Lebzeiten teilweise heftigst \\ umstritten. Das galt erst recht für die Zeit nach seinem Tod. Zwar dominierte keynesianisches \\ Gedankengut die Wirtschaftspolitik der Nachkriegszeit. Doch spätestens seit den 1970er \\ Jahren begann deren intellektuelle Anziehungskraft angesichts gleichzeitig steigender \\ Arbeitslosigkeit, Staatsverschuldung und Inflation merklich zu schwinden. In jüngster Zeit ist \\ allerdings eine Trendwende erkennbar, die mit Fehlern der Wirtschaftspolitik, aber auch mit \\ neuen Herausforderungen im Zusammenhang steht.
}

\begin{abstract}
Es begann schon vor gut zehn Jahren. In der heraufziehenden Finanzmarktkrise in den Jahren 2007 und 2008 stand die Wirtschaftspolitik in allen größeren Volkswirtschaften vor der Herausforderung, Maßnahmen gegen die massiven Belastungen zu ergreifen, die sich aus der bedrohlichen Schieflage und dem teilweisen Zusammenbruch des globalen Finanzsystems auftürmten. Im Unterschied zu den angelsächsischen Ländern, denen dies bei allen auch dort üblichen Auseinandersetzungen deutlich leichter von der Hand ging, tat sich die Wirtschaftspolitik in Deutschland besonders schwer.
\end{abstract}

Das lag an dem Kurs, der mit breiter Unterstützung in allen größeren Parteien zuvor eingeschlagen worden war und der von einer prinzipiellen wirtschaftspolitischen Enthaltsamkeit des Staates ausging. Diese Haltung fand seinerzeit inren deutlichsten Ausdruck im „Hamburger Appell“ (Funke et al., 2005), der von gut 250 teilweise prominenten Ökonominnen und Ökonomen wie Hans-Werner Sinn unterzeichnet wurde. In diesem Appell heißt es: „Die

(C) Der/die Autor:in(nen) 2021. Open Access: Dieser Artikel wird unter der Creative Commons Namensnennung 4.0 International Lizenz veröffentlicht (creativecommons.org/licenses/by/4.0/deed.de).

Open Access wird durch die ZBW - Leibniz-Informationszentrum Wirtschaft gefördert.

Prof. Dr. Gustav A. Horn ist apl. Professor an der Universität Duisburg-Essen und war wissenschaftlicher Direktor des Instituts für Makroökonomie und Konjunkturforschung (IMK) in der Hans-Böckler-Stiftung in Düsseldorf. Er ist Vorsitzender der Keynes-Gesellschaft. gesamtwirtschaftliche Nachfrage ist eine bedeutende und komplex strukturierte ökonomische Größe, die sich einer nachhaltigen Steuerung weitestgehend entzieht." Dies war als eine klare Aussage gegen jedwede Form keynesianischer Wirtschaftspolitik gemeint. Der Text, im Vorfeld der Bundestagswahl 2005 entstanden, bestreitet schlichtweg die Möglichkeit, die Wirtschaft über eine Stimulanz der gesamtwirtschaftlichen Nachfrage zu stabilisieren. Keynes wurde in Deutschland auch wirtschaftspolitisch für tot erklärt.

\section{Die stille Wiedergeburt}

Nur wenige Jahre später änderten sich die Einstellungen vor dem Hintergrund der Finanzmarktkrise grundlegend. Dies galt weniger für die akademischen wirtschaftspolitischen Debatten, die in Deutschland unverändert sehr stark auf die Angebotsseite fokussiert waren. Für sie waren allein strukturelle Reformen, die insbesondere in Deregulierungen am Arbeitsmarkt und Kürzungen von Ansprüchen an die Sozialversicherung bestehen, maßgeblich, um wirtschaftliches Wachstum zu erreichen. Der Sachverständigenrat (SVR) sorgte sich 2007, als sich in den USA die Finanzmarktkrise allmählich zu entfalten begann, vor allem darum, dass in Gegenwart einer positiven Konjunkturdynamik Arbeitsmarktreformen in Deutschland zurückgenommen werden könnten (SVR, 2007). Folglich stellte er sein Jahresgutachten unter das Motto: Das Erreichte nicht verspielen.

Nur ein weiteres Jahr später, als die Finanzmarktkrise dann die globalen Märkte erreicht hatte, und die Wirtschaftspolitik gefordert war, stellte sich in Deutschland sowohl in den meisten beratenden ökonomischen Forschungsinstituten als auch in der Politik eine gewisse Ratlosigkeit ein. Wie sollte man mit den vorherrschenden Konzepten dieser Krise begegnen, wenn die gängigen 
makroökonomischen Modelle, die von einer inhärenten Stabilität der Märkte ausgingen, eine solche Krise gar nicht kannten und folglich auch keine Gegenmaßnahmen. Dies kam in einer Erklärung des damaligen DIW-Präsidenten Zimmermann in seinem Plädoyer für einen Prognoseverzicht deutlich zum Ausdruck: „Man muss auch mal zugeben können, dass man nichts weiß“" (DIW, 2010). Aus dieser Sicht waren die Empfehlungen Prognoseverzicht und Abwarten vor dem Hintergrund der sich entfaltenden Krise konsequent. Sie zeigten auch, dass vielen wirtschaftspolitischen Berater:innen die Kenntnisse für den Umgang mit einem Einbruch der gesamtwirtschaftlichen Nachfrage, wie er im Gefolge der Finanzmarktkrise zu erkennen war, abhanden gekommen waren.

Wusste man wirklich nichts? Wohl kaum. In seiner aufsehenerregenden Presidential Address 2006 hatte der Nobelpreisträger George Akerlof (Akerlof, 2007) neuere Erkenntnisse aus der mikroökonomischen Theorie, wie der Verhaltensökonomie, in die Makroökonomie übertragen. Eines seiner zentralen Ergebnisse war, dass auf der Basis neuerer Erkenntnisse in der Mikroökonomie, die auf in Experimenten überprüften ökonomischen Verhalten Einzelner basieren, gesamtwirtschaftlich eine Stabilisierung durch wirtschaftspolitische Eingriffe möglich ist (Akerlof, 2007 , 8). Damit gab es in der akademischen Ökonomie auch seinerzeit sehr wohl neuere fundierte Begründungen für eine Stabilisierungspolitik, die aber in Deutschland in der wirtschaftspolitischen Beratung nicht hinreichend zur Kenntnis genommen wurden. Für die wirtschaftspolitischen Akteure war während der Finanzmarktkrise Abwarten in der Erwartung eines massiven Anstiegs der Arbeitslosenzahlen, der nach dem dramatischen Einbruch der Wirtschaftsaktivität im Sommer 2008 unvermeidlich erschien, keine Option. Da die neueren Theorien noch nicht rezipiert waren, griff man instinktiv auf ältere, wirtschaftspolitisch vermeintlich längst überholte Konzepte zurück. Dies war die stille wirtschaftspolitische Wiedergeburt von John Maynard Keynes.

Sie war still, weil kein maßgeblicher Politiker und keine Politikerin sich offen zu einer keynesianischen Politik bekennen wollte. Schließlich war diese zuvor teilweise von den gleichen Personen jahrelang als unwirksam oder sogar als schädlich bezeichnet worden, weil sie den notwendigen Strukturwandel behindere. Der SVR bezeichnete in seinem Jahresgutachten 2008, diese Politik denn auch verschämt als „konjunkturgerechte Wachstumspolitik" (SVR, 2008, Ziff. 20 ff.) und legte damit eine bei vielen willkommene Sprachregelung vor, die einen unveränderten wirtschaftspolitischen Kurs suggerieren sollte.

Es war dennoch eine Wiedergeburt keynesianischen Denkens. Denn die von der damaligen Bundesregierung beschlossenen Maßnahmen entsprachen weitgehend keynesianischem Lehrbuchdenken (Kromphardt, 2020, 150 f.). Sie wirkten vor allem über die Nachfrageseite, deren Einbruch die Wirtschaftspolitik schließlich zum Handeln trieb. Es begann mit dem Konjunkturpaket I, das, wenn auch finanziell mit knapp 12 Mrd. Euro eher bescheiden ausgestattet, die faktische Kehrtwende in der Wirtschaftspolitik einleitete. Denn zum ersten Mal seit den 1980er Jahren wurde ein diskretionäres Konjunkturprogramm auf den Weg gebracht. Das bedeutet, man verließ sich fiskalpolitisch nicht wie zuvor im Einklang mit dem Washington Consensus in der Stabilisierungspolitik üblich auf die Wirkung der automatischen Stabilisatoren, sondern steuerte durch explizit beschlossene höhere Ausgaben und niedrigere Steuern gegen den Nachfrageeinbruch. Damit folgte man eindeutig keynesianischen Überlegungen. Bemerkenswert war, dass die Förderung von Kurzarbeit, einer Arbeitsmarktmaßnahme, Bestandteil des Pakets war. Damit stabilisierte man direkt die Einkommen der Beschäftigten, die ansonsten arbeitslos geworden wären. Deshalb gelang es in Deutschland im Unterschied zu anderen Volkswirtschaften den Konsum vergleichsweise stabil zu halten.

Dieses Vorgehen setzte sich mit dem Konjunkturpaket II verstärkt fort, das finanziell wesentlich umfangreicher und mit einem höheren Investitionsanteil versehen war. Man betrieb keynesianische Stabilisierungspolitik, ohne dass man es eigentlich wollte. In der Öffentlichkeit wurde dies jedoch zu Recht als die „Rückkehr des Meisters“ (Skidelsky, 2009) verstanden. Gleichzeitig gerieten die vorherrschenden wirtschaftspolitischen Konzepte wegen ihrer offenkundigen Nicht-Anwendbarkeit während einer Krise massiv unter Druck (Colander et al., 2009).

\section{Der nicht lineare Paradigmenwechsel}

Trotz dieser markanten Kehrtwende im wirtschaftspolitischen Handeln während der Finanzmarktkrise kann in diesen Jahren von einem nachhaltigen Paradigmenwechsel in Richtung einer keynesianischen Wirtschaftspolitik nicht die Rede sein. Dies zeigte sich primär in der nachfolgenden Krise des Euroraums. Zwar gab es, und dies schon seit Jahren, in vielen Mitgliedstaaten wie Griechenland Probleme auf der Angebotsseite durch zu hohe Inflationsraten. Aber die Finanzmarktkrise hatte zudem auch eine Nachfrageschwäche erzeugt (Horn, 2015). Beschlossen wurden jedoch nur angebotsfördernde Maßnahmen verbunden mit einer teilweise überaus restriktiven Nachfragepolitik. Das Ergebnis waren tiefe Rezessionen mit hoher Arbeitslosigkeit.

Diese wirtschaftspolitische Strategie wurde vor allem von der deutschen Bundesregierung auf europäischer Ebene 
gefordert und forciert. Auch im Inland wurde mit der Einführung der Schuldenbremse 2009 eine klare Absage an keynesianische Wirtschaftspolitik erteilt. Keynesianische Politik wurde schließlich im öffentlichen Diskurs immer noch als eine Politik des Schuldenmachens interpretiert, auch wenn dies nur für Krisenlagen stimmt. Man könnte die Schuldenbremse auch als eine kodifizierte keynesianische Politik verstehen, die einerseits in einer Krise die Schuldenaufnahme erlaubt, die Politik aber andererseits zwingt, diese in Zeiten guter Konjunktur auch wieder abzubauen. Doch ist dies eine sehr wohlwollende Interpretation des beschlossenen Mechanismus. Dessen ganze Konstruktion ist darauf ausgerichtet, Stabilisierungspolitik zu erschweren. Auf die schwerwiegenden Folgen haben verschiedene Autor:innen immer wieder hingewiesen (Rietzler und Truger, 2019).

Schließlich hat die Regierung erst ab einem zu erwartenden Einbruch von tiefer als $2 \%$ freie Hand für Konjunkturprogramme. Für die anschließende Zeit gibt es restriktive Regelungen zum Abbau der aufgelaufenen Schulden, die eine beginnende Erholung erschweren können. Aber auch bei milderen und vor allem längerwährenden Rezessionen kann die Arbeitslosigkeit spürbar steigen. In diesem Fall dürfen entsprechend des Washington Consensus im Rahmen der Schuldenbremse dennoch nur die automatischen Stabilisatoren wirken. Umgekehrt gilt, dass in Zeiten guter Konjunktur sogar ein relativ großer Spielraum besteht, weiterhin die Ausgaben hoch zu halten (Rietzler und Truger, 2019). D. h. die Schuldenbremse weist in hohem Ausmaß prozyklische Eigenschaften auf und steht damit im Gegensatz zur keynesianischen Lehre.

Doch war das Scheitern des Washington Consensus in diesen krisenbehafteten Zeiten angelegt. In ihrer wegweisenden Publikation wiesen Blanchard und Leigh (2013) nach, dass die Wirkungen der restriktiven Finanzpolitik während der Eurokrise fortwährend unterschätzt wurden. Viele andere Publikationen mit ähnlichen Resultaten folgten mit dem Ergebnis: Die restriktive Nachfragepolitik war zu hart und resultierte in nicht zu rechtfertigender Arbeitslosigkeit. Damit war die Sichtweise, Nachfragepolitik sei als wirtschaftspolitisches Instrument von Stabilisierungspolitik weitgehend überflüssig, fundamental erschüttert.

\section{In der Pandemie regiert Keynes}

Wie ausgeprägt die tektonische Verschiebung der wirtschaftspolitischen Ansichten war, zeigte sich gleich zu Beginn der Corona-Pandemie. Im Gegensatz zur Finanzmarktkrise gab es keinen Grundsatzstreit mehr über die Notwendigkeit von Stabilisierungspolitik auch über die Nachfrageseite. Dies zeigte sich vor allem in dem Konjunkturprogramm, das die daniederliegende Binnennachfrage nach dem ersten Lockdown stimulieren sollte. Es enthält mit der Senkung der Mehrwertsteuer ein explizites Element, das die Konsumnachfrage anregen soll. In die gleiche Richtung, nur direkter, wirkt der Kinderbonus, der unmittelbar die Einkommen von Familien und damit deren Nachfrage erhöht. Darüber hinaus werden über Steuererleichterungen sowie Zuweisungen an Länder und Kommunen Investitionen gefördert. Schließlich wurde wie schon in der Finanzmarktkrise die Möglichkeit zu Kurzarbeit ausgeweitet, um Beschäftigung und Einkommen der Arbeitenden in vom Lockdown betroffenen Unternehmen zu stabilisieren.

Die Maßnahmen gehen aber deutlich über jene in der Finanzmarktkrise hinaus. In krassem Gegensatz zum früheren Vorgehen wird in dieser Krise keine restriktive, sondern nicht zuletzt auf Initiative der Bundesregierung eine markant expansive Nachfragepolitik auf EU-Ebene betrieben. Mit der Einrichtung des Recovery Funds wird die Verantwortung für die Stabilisierungspolitik sogar auf die EU-Ebene verlagert. Damit wird faktisch anerkannt, dass die EU als ein gemeinsamer Markt auch einer aggregierten Nachfragepolitik in Krisenzeiten bedarf.

Weitere Elemente kommen durch die Besonderheit der Krise hinzu. Die Ansteckungsgefahr durch das Virus erforderte, dass sozialer Konsum weitgehend reduziert werden musste. Die Schließung von Einzelhandelsgeschäften, Restaurants etc. kann als eine Art negativer Angebotspolitik interpretiert werden. Dies ist für sich genommen zwar nicht keynesianisch, löst aber eine keynesianische Schwäche aus. Wenn die Umsätze der Unternehmen qua Verordnung auf null gesetzt werden, brechen in diesen Bereichen nicht nur Investitionen und die Beschäftigung ein, was schon alleine eine schwächere Gesamtnachfrage bedeutet. Vielmehr verursacht ein solcher Schock auch gesamtwirtschaftlich eine tiefe Verunsicherung sowohl bei den Unternehmen als auch bei den Konsumenten aus. Der Börsencrash und die völlig irrationalen Hamsterkäufe im Frühjahr 2020 waren deutliche Hinweise hierauf. Dies rückt einen anderen und lange Zeit viel zu wenig beachteten Aspekt der keynesianischen Ökonomie in den Mittelpunkt: Unsicherheit.

\section{Die verlorene Unsicherheit}

Mit Unsicherheit als einer zentralen Kategorie der keynesianischen Lehre im Fokus lohnt sich ein genauerer Blick auf die Dynamik des Paradigmenwechsels. Er lässt erkennen, dass der Wandel der wirtschaftspolitischen Einstellungen keinesfalls eine schlichte Rückkehr zu einem Keynesianismus ist, wie er in den frühen 1970 Jahren vor dem Aufstieg neoliberaler oder neuklassischer Vorstellungen gängig war. Denn zu jener Zeit wies die vorherrschende Variante des Keynesianismus im Vergleich zum ursprünglichen Keynesianismus ein merklich verengtes Blickfeld auf. 
Der Kern keynesianischer Lehre beschränkte sich in jener Zeit auf das Wirken von Preis-Lohn-Mechanismen (Malinvaud, 1977). Ihnen wurde eine zu geringe Flexibilität oder zu große Trägheit attestiert, um jederzeit die Märkte im Gleichgewicht zu räumen. Die theoretischen Überlegungen zeigten, dass dabei auch eine Konstellation entstehen kann, bei der sich durch gemessen an den Markträumungserfordernissen zu niedrigen Reallöhnen Unterbeschäftigung aufgrund von Nachfragemangel bildet. Dies wird als Regime keynesianischer Unterbeschäftigung interpretiert. In diesem Fall tragen Nachfrageprogramme zu einer höheren Beschäftigung und damit zur wirtschaftlichen Stabilisierung bei. Der Vorteil dieser Modelle, die in ihrer Summe als neo- oder neukeynesianisch bezeichnet werden, ist, dass sie aus einzelwirtschaftlichem Verhalten hergeleitet werden können. Daraus leitet sich die wichtige Erkenntnis ab, dass gesamtwirtschaftliche Ungleichgewichte sehr wohl das Ergebnis einzelwirtschaftlich rationaler Verhaltensweisen sein können. Damit sahen sich die Vertreter dieser keynesianischen Modellklassen auf Augenhöhe mit den Vertretern der neuklassischen Makroökonomie, die die einzelwirtschaftliche Fundierung ihrer Modelle als Merkmal theoretischer Überlegenheit verstanden (Lucas, 1981), da Keynes seine theoretischen Überlegungen nicht oder bestenfalls anekdotisch mit einzelwirtschaftlichem Verhalten begründete. Die neukeynesianischen Modelle schlossen dieses Lücke.

Doch diese Errungenschaft führte im Grunde von Keynes weg. Die Unterbeschäftigungsgleichgewichte sind hier ausschließlich das Resultat träger Preis- und Lohnbewegungen. Ohne diese Trägheit wären die Märkte im Gleichgewicht. Man kann aus guten Gründen daran zweifeln, ob Keynes dies auch so gesehen hätte (Skidelsky, 2009). Denn in dieser Sichtweise fehlt Unsicherheit als einer der Tragpfeiler keynesianischen Denkens. Damit liegt der Neukeynesianismus nicht weit von der Neuklassik entfernt, die die Rolle träger Preis- und insbesondere Lohnanpassungen ähnlich sieht. Deren Flexibilisierung wäre demnach in beiden Sichtweisen die naheliegende Möglichkeit, ein markträumendes Gleichgewicht herzustellen.

Dies ist jedoch strittig, zumindest unter Keynesianern. Auf der einen Seite stehen jene, die genau in diese Richtung tendieren (Mankiw, 1985). Sie gehörten zu den Vorreitern des Washington Consensus, der die Unterschiede zwischen den beiden Denkschulen auf wirtschaftspolitischer Ebene aufweichte. Auf der anderen Seite stehen aber jene, die flexible Preis- und Lohnmechanismen weder für möglich noch für wünschenswert halten (Akerlof und Yellen, 1985). Die Inflexibilität gemessen an den Erfordernissen zur Markträumung hat in dieser Sichtweise ihre rationalen Gründe, die sowohl einzel- als auch gesamtwirtschaftlicher Natur sind. Sie bestehen letztlich in der Unsicherheit der Märkte und den Kosten der Informationsgewinnung.
Dies führt zu dem maßgeblichen Treiber des Paradigmenwechsels: Dem Umgang mit Unsicherheit und damit der Rückkehr zum ursprünglichen Keynes.

\section{Die Rückkehr des alten Meisters}

Es geht also um Unsicherheit. Sie spielt eine zentrale Rolle im keynesschen Denken (Skidelsky, 2009). Insbesondere unkalkulierbare, also nicht mit einer Wahrscheinlichkeit versehbare Risiken sieht er als eine Bedrohung wirtschaftlicher Stabilität an. In diese Kategorie gehören für inn die Risiken auf wenig oder unregulierten Finanzmärkten. Das aber steht in krassem Gegensatz zu der Anschauung, die bei den meisten Ökonom:innen bis zur Finanzmarktkrise vorherrschte (Skidelsky, 2009). Sie sahen die Risiken auf Finanzmärkten als kalkulierbar und damit als absicherbar an. Hierfür gab es und gibt es vermeintlich wirksame Finanzmarktinstrumente. Für diese Sichtweise sprach, dass es seit dem Zweiten Weltkrieg keine dramatischen Finanzmarktkrisen gegeben hatte. Nicht berücksichtigt wurde, dass die Abwesenheit von Krisen weitgehend auf den Erfahrungen aus der Depression der 1930er Jahre und den Lehren für strikte Regulierungsmaßnahmen, die insbesondere aus keynesianischer Sicht hieraus gezogen wurden, basierte. Vor diesem Hintergrund ist es nicht verwunderlich, dass unkalkulierbare Unsicherheit aus dem makroökonomischen Denken allmählich verschwand und keinen Eingang in die quantitativen Modelle der jüngeren Zeit fand. Das gilt auch für die neukeynesianischen Modelle.

Die Finanzmarktkrise hat all diese Sichtweisen widerlegt. Zugleich bestätigte sie damit Keynes Zugang zur Ökonomie. Inmitten der ab dem Sommer 2008 zusammenbrechenden Produktion und den davon ausgehenden Gefahren für die Beschäftigung ergriff diese Unsicherheit die Wirtschaftspolitik in Deutschland. In den ihnen unbekannten Gewässern der Finanzkrise tat sich für die wirtschaftspolitisch Handelnden eine Untiefe auf, deren Grund für sie nicht erkennbar war. Die üblichen Ansätze boten keinen Halt. Allein die keynessche Lehre hielt dagegen Instrumente in Gestalt von Konjunkturprogrammen bereit, die Rettung versprachen. Sie zeigten denn auch auf zweifache Weise Wirkung. Zum einen stabilisierten die erhöhten Ausgaben des Staates die Wirtschaft direkt. Mindestens ebenso wichtig waren aber zum anderen die indirekten Effekte über die Erwartungen. Indem mittels Kurzarbeit die Beschäftigung nur vergleichsweise geringfügig zurückging und die Arbeitslosigkeit gemessen an dem dramatischen Produktionseinbruch nur geringfügig stieg, blieben die Einkommenserwartungen der Konsumenten relativ stabil. Es kam folglich nicht zu einem Angstsparen, das den Konsum hätte einbrechen lassen und so die Krise verschärft hätte.

Ähnliches gilt für die Unternehmen. Allein die Beschlüsse über die Abwrackprämie und insbesondere die Investitionsvorhaben hellten die Erwartungen der Unternehmen 
auf, sodass sie auf einen Beschäftigungsabbau weitgehend verzichteten und sich ihre Investitionsaktivität innerhalb kurzer Zeit stabilisierte. Das hat die Dauer der Krise stark verkürzt. Die positive Wirkung trat ein, noch bevor viele Mittel aus den Investitionsprogrammen überhaupt geflossen waren. Das zeigt, wie wichtig die Erwartung einer Stabilisierung für die Stabilisierung ist. Genau dies ist Keynes pur.

Diese Erfahrung wiederholt sich nun in anderer Form, aber wesentlich reibungsloser während der Corona-Pandemie. Die Schließung weiter Teile der Wirtschaft bei Ausbruch der Pandemie musste zwangsläufig ebenfalls eine tiefe Verunsicherung auslösen. Da die Schließung jedoch ein negativer Angebotsschock ist, konnte eine konventionelle keynesianische Nachfragepolitik nicht die einzige Antwort sein. Die rasch beschlossenen Überbrückungsgelder können denn auch als eine Form keynesianischer Angebotspolitik beschrieben werden. Sie unterstützen zwar für keynesianische Programme eher unüblich die Angebotsseite, aber ihr tieferer Zweck ist es, die Unsicherheit zu mindern. Genau das ist keynesianisches Handeln in Krisensituationen. Nachfrageprogramme ergänzen dieses Vorgehen. Dies wiederholt sich bei jeder Welle, sodass wirtschaftliche Risiken durch die Unterstützung des Staates mehr oder minder begrenzt werden.

In dieser Krise kommen noch zusätzliche Maßnahmen zum Zuge, die über den traditionellen Keynes hinausgehen. Die globale Wirtschaft steht mitten in einem grundlegenden Wandel. Digitalisierung und der Übergang zu nachhaltigen Produktionsweisen erfordern massive Investitionen sowohl im privaten wie im öffentlichen Sektor. Diesen Wandel zu unterstützen, sogar zu beschleunigen, sowie gleichzeitig die Wirtschaft zu stimulieren, ist das Ziel zahlreicher Maßnahmen, die während dieser Pandemie ergriffen wurden. Sie bekämpfen damit Unsicherheit doppelt. Denn neben der konjunkturellen Belastung erzeugt auch der Wandel der Wirtschaft Ängste um Beschäftigung und Einkommen. Beides wird auf diese Weise adressiert.

\section{Keynes ist wieder da}

Die grundlegende Erfahrung der vergangenen Jahre ist, dass die inhärente Unsicherheit der Märkte, insbesondere der Finanzmärkte, jederzeit zum Tragen kommen kann. John Maynard Keynes ist der Ökonom, der sich am intensivsten mit den Folgen und der Bekämpfung von Unsicherheit auseinandergesetzt hat. Insofern ist seine wirtschaftspolitische Wiederentdeckung kein Zufall, sondern nahezu zwangsläufig. Keynes grenzt sich damit scharf von jenen Sichtweisen ab, die das wirtschaftspolitische Handeln während der vergangenen Jahrzehnte geprägt haben. Denn diese, und das gilt einschließlich des Neukeynesianismus, sehen fundamentale Unsicherheit nicht als ein zentrales Thema, und das ist sogar verständlich. Schließlich war die wirtschaftliche Entwicklung der Nachkriegszeit zumindest in Deutschland weitgehend frei von dramatischen Krisen. Dass dies nicht zuletzt auf den keynesianischen Lehren aus der Depression der 1930er Jahre beruhte, machte eines deutlich: Wer Keynes vernachlässigt, wird am Ende reumütig zu ihm zurückkehren.

\section{Literatur}

Akerlof, G. A. (2007), The Missing Motivation in Macroeconomics, American Economic Review, 97(1), 5-36.

Akerlof, G. A. und J. L. Yellen (1985), A Near-rational Model of the Business Cycle, with Wage and Price Inertia, The Quarterly Journal of Economics, 100(5), 823-838, doi:10.1093/qje/100.Supplement.823.

Blanchard O. und D. Leigh (2013), Growth Forecast and Fiscal Multiplier, American Economic Review, 103(3), 117-120).

Colander D., M. Goldberg, A. Haas, K. Juselius, A. Kirman, T. Lux und B. Sloth (2009), The Financial Crisis and the systemic Failure of the Economomics Profession, Critical Review, 21(2-3), 249-267.

DIW (2010), Presseerklärung, 6. August, https://www.diw.de/de/ diw_01.c.359083.de/zimmermann_verteidigt_prognoseverzicht.html (17. März 2021)

Funke, M., B. Lucke und T. Straubhaar (2005), Hamburger Appell, https:// www.hwwi.org/uploads/tx_wilpubdb/Hamburger_Appell.pdf (8. März 2021).

Horn, G. A (2015), Wirtschaftliche Krisen bewältigen, Springer Gabler.

Kromphardt, J. (2020), John Maynard Keynes, München (utb).

Lucas, R. E. (1981), Studies in Business-Cycle Theory, Cambridge London.

Malinvaud, E. (1977), The Theory of Unemployment Reconsidered, Basil Blackwell.

Mankiw, N. G. (1985), Small Menu Costs and Large Business Cycles: A Macroeconomic Model of Monopoly, The Quarterly Journal of Economics, 100(2), 529-538, doi:10.2307/1885395.

Rietzler, K. und A. Truger (2017), Die Schuldenbremse als Ursache der deutschen Konsolidierungserfolge? Eine vergleichende Analyse der strukturellen Konsolidierung der öffentlichen Haushalte der Gebietskörperschaften von 1991 bis 2016, in H. Hagemann, J. Kromphardt und M. Marterbauer (Hrsg.), Keynes, Geld und Finanzen, Schriften der KeynesGesellschaft, Bd. 11, Metropolis, 331-353.

Sachverständigenrat zur Begutachtung der gesamtwirtschaftlichen Entwicklung (SVR, 2007), Das Erreichte nicht verspielen, Jahresgutachten 2007/2008.

Sachverständigenrat zur Begutachtung der gesamtwirtschaftlichen Entwicklung (SVR, 2008), Die Finanzkrise meistern - Wachstumskräfte stärken, Jahresgutachten 2008/2009.

Skidelsky, R. (2009), Die Rückkehr des Meisters, Kunstmann München.

Title: The Repressed Keynes

Abstract: This month marks the 75th anniversary of the death of John Maynard Keynes. He was one of the most important economists of the 20th century, if not of any century. Yet his teachings and advice were already highly controversial during his lifetime. This applied even more after his death. Keynesian ideas dominated economic policy in the postwar period. But since the 1970s at the latest, their intellectual appeal began to wane noticeably in the face of concurrently rising unemployment, national debt and inflation. Recently, however, a trend reversal has become apparent, which is related to mistakes in economic policy as well as to new challenges. 